\title{
Influence of substrate additives on the mechanical properties of ultrathin oxide coatings on poly(ethylene terephthalate)
}

\author{
G. Rochat ${ }^{\mathrm{a}}$, Y. Leterrier ${ }^{\mathrm{a}}$, P. Fayet ${ }^{\mathrm{b}}$, J.-A.E. Månson ${ }^{\mathrm{a}, *}$ \\ ${ }^{\mathrm{a}}$ Laboratoire de Technologie des Composites et Polymères (LTC), Ecole Polytechnique Fédérale de Lausanne (EPFL), CH-1015 Lausanne, Switzerland \\ ${ }^{\mathrm{b}}$ Tetra Pak (Suisse) SA, Tetra Pak R\&D, Plasma Technology, C.P. 32, CH-1680 Romont, Switzerland
}

Received 29 April 2004; accepted in revised form 18 March 2005

Available online 13 May 2005

\begin{abstract}
The mechanical properties of ultrathin silicon oxide $\left(\mathrm{SiO}_{x}\right)$ coatings plasma-deposited on poly(ethylene terephthalate) (PET) films were investigated with particular attention paid to the effect of additives located in the superficial layers of the polymer substrate. The cohesive and adhesive properties of the thin oxide coating were derived from the analysis of fragmentation tests carried out in situ in a scanning electron microscope. The cohesive strength of the coating was determined assuming a Weibull probability of failure of the oxide, and the coating/ substrate interfacial shear strength (IFSS) was calculated by means of a stress transfer analysis with a perfectly plastic interface. It was shown that the presence of additives in the superficial layers of PET substrates leads to a $20 \%$ decrease of the crack onset strain, which is due to an increase of the coating defect density, as revealed by means of atomic oxygen etching. The stress concentration induced by coating microdefects was modeled, and was shown to induce a decrease in the cohesive properties of the coating, which correlates with the observed decrease of crack onset strain. Moreover, the adhesion was found to be very high, with a IFSS higher than the substrate bulk shear stress at yield, irrespective of the presence of additives.
\end{abstract}

(C) 2005 Elsevier B.V. All rights reserved.

Keywords: $\mathrm{SiO}_{x}$; PET; Additives; Cohesion; Adhesion; Fragmentation; Defects

\section{Introduction}

Plasma-deposited oxide and nitride thin coatings on polymer substrates have emerged in the past decade as gasbarrier composite materials for food and pharmaceutical packaging [1]. Layered materials based on such thin film structures enable extremely low gas permeability, which could be suitable for advanced applications in the field of polymer-based displays [2]. A number of recent studies have shown that the defect structure of the coating control the oxygen and water vapor permeability of the coated film $[3,4]$, as also its mechanical behavior such as cohesive and adhesive properties [5-8]. Nanosized oxide coatings are characterized by the presence of two types of defects, namely microdefects and nanodefects [3]. Microdefects,

\footnotetext{
* Corresponding author. Tel.: +4121693 4281; fax: +41216935880

E-mail address: jan-anders.manson@epfl.ch (J.-A.E. Månson).
}

which comprise pinholes and microcracks are mainly created by plasma arching due to dielectric variations in the plasma environment. Nanodefects, on the other hand, result from the non-equilibrium $\mathrm{SiO}_{x}$ formation, and correspond to local stoichiometric variations in the ideal $\mathrm{SiO}_{2}$ network. In fact, in cases where they are larger than the oxygen molecule diameter, and percolate through the entire coating thickness, nanometric defects affect coating permeability more than micrometric defects covering the same surface [9]. Moreover, flaws or cracks in the coating lead to stress concentrations as soon as the film is mechanically stressed. It is well-known that a maximal stress concentration of 3 occurs at the equator of a circular hole in a thin elastic plate under tension [10]. Sharper defects give rise to much larger stress concentrations. For an elliptic hole of major axis $a_{1}$ and minor axis $a_{2}$ in a thin plate, the classic result states that the stress concentration $\sigma_{\max } / \sigma_{\infty}=1+2\left(a_{1} /\right.$ $a_{2}$ ), where $\sigma_{\max }$ and $\sigma_{\propto}$ represent the maximum stress and the far-field applied stress, respectively. This diverges as 
$a_{2} \rightarrow 0$. In other words, sharp microcracks such as those responsible for stress relaxation processes during coating growth are critical sources for damage initiation.

A further source of coating defects include the presence of inorganic additives in the superficial layers of the polymer substrate, such as anti-block particles [11]. These additives protrude on the polymer surface and increase the coating roughness [12-14], and are also likely to affect the coating microstructure in the early stages of the deposition process. Whereas additive-induced roughness marginally affects the mechanical properties of the coating [5], a modification of the microstructure and defect population of the coating is expected to significantly influence the mechanical properties of the coated system.

The present work analyses the effect of substrate additives on the cohesive and adhesive properties of thin silicon-oxide based coatings deposited by plasma enhanced chemical vapor deposition (PECVD) on semi-crystalline poly(ethylene terephthalate) (PET) substrates. To this end, the mechanical properties of the coating determined by means of in-situ in a scanning electron microscope fragmentation tests [15] were analyzed using defect size distributions measured with a reactive ion etching method [16] and analytical calculations of stress concentrations at defect sites.

\section{Experimental methods}

\subsection{Materials}

Thin silicon oxide coatings $\left(\mathrm{SiO}_{x}\right)$ were deposited by plasma enhanced chemical vapor deposition (PECVD) from hexamethyldisiloxane vapor (HMDSO) on semi-crystalline poly(ethylene terephthalate) (PET) substrates. Two $12 \mu \mathrm{m}$ thick PET substrates of the same composition were investigated, one containing chemical additives in superficial layers, that form irregular aggregates of typical
Table 1

Oxygen transmission rate (OTR) and roughness $\left(R_{\mathrm{a}}\right)$ of uncoated and $\mathrm{SiO}_{x}$ coated PET films

\begin{tabular}{llllll}
\hline PET substrate & Uncoated & & $\mathrm{SiO}_{x}$ coated $(10 \mathrm{~nm})$ \\
\cline { 2 - 3 } & $\begin{array}{l}\text { OTR }\left[\mathrm{cm}^{3}(\mathrm{STP}) /\right. \\
\left.\mathrm{m}^{2} / \text { day } / \mathrm{atm}\right]\end{array}$ & $\begin{array}{l}R_{\mathrm{a}} \\
{[\mathrm{nm}]}\end{array}$ & $\begin{array}{l}\text { OTR }\left[\mathrm{cm}^{3}(\mathrm{STP}) /\right. \\
\left.\mathrm{m}^{2} / \mathrm{day} / \mathrm{atm}\right]\end{array}$ & $\begin{array}{l}R_{\mathrm{a}} \\
{[\mathrm{nm}]}\end{array}$ \\
\hline Without additives & $111.9 \pm 5.8$ & 0.5 & & $0.9 \pm 0.1$ & 0.7 \\
With additives & $114.2 \pm 4.2$ & 1.6 & & $1.2 \pm 0.3$ & 1.6 \\
\hline
\end{tabular}

diameter of a few hundreds of nanometers, and the other being free of these additives. Atomic force micrographs (AFM) of the PET surfaces are reproduced in Fig. 1. The thickness of the $\mathrm{SiO}_{x}$ coating was found to be equal to 10 $\mathrm{nm}$ from X-ray fluorescence measurements. The oxygen transmission rate (OTR) of the two types of films, with and without the oxide coating, was measured using an oxygen permeation cell (Mocon OX-TRAN $\AA$, Minneapolis) at ambient temperature on $10 \mathrm{~cm}^{2}$ circular samples, with a resolution of $0.1 \mathrm{~cm}^{3} / \mathrm{m}^{2} /$ day, and their mean roughness was measured from the AFM amplitude signal on $1 \times 1 \mu^{2}$ zones. The results are summarized in Table 1 . The presence of additives clearly increases the roughness of the PET surface, whereas it does not affect the OTR of the bare polymer. In contrast, both roughness and OTR of the coated PET are higher when additives are present, which suggests that the additives increased the density of defects in the oxide layer.

\subsection{Atomic oxygen etching}

Accurate characterization of the defect population of the coating is important in order to discriminate the respective influence of different parameters, such as plasma gas fluxes, substrate surface morphology, and substrate additives, on the coating cohesion and coating/substrate interfacial adhesion. Advanced microscopy techniques (SEM, TEM, AFM) appear to be well suited for this purpose, but they are tedious, and very time consuming. Alternatively, Wer- (a)

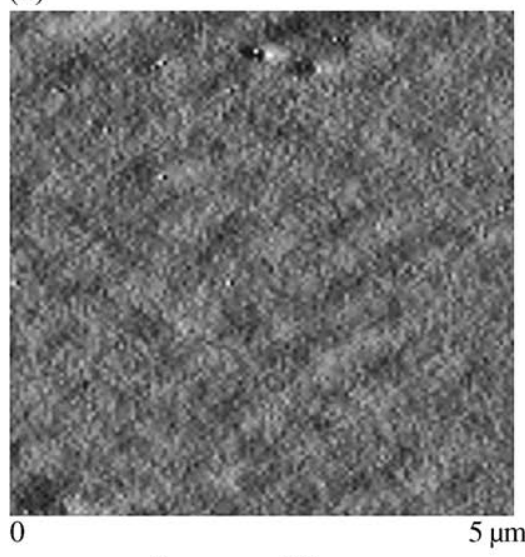

Z-range: $30 \mathrm{~nm}$ (b)

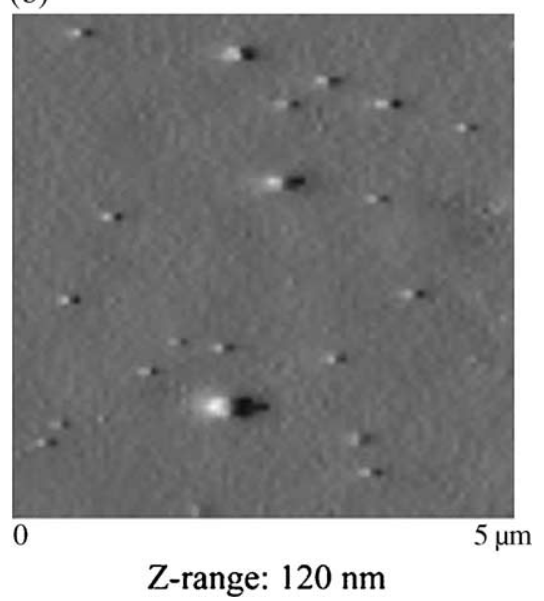

Fig. 1. AFM micrographs of PET without additives (a) and with additives (b). 
theimer and coworkers developed a new technique for obtaining the defect density and size distribution of thin silicon oxide coatings on polymers [16]. This method is based on the progressive etching of the polymer substrate exposed to atomic oxygen, at the location of coating defects. Atomic oxygen (AO) plasmas are well-known for their capacity to modify the superficial structure of semicrystalline polymers, whereas they do not affect silicon oxide layers [16]. The stability of silicon oxide in the presence of $\mathrm{AO}$ was utilized to determine the presence and size of defects in the thin $\mathrm{SiO}_{x}$ coatings, which otherwise would be extremely difficult to observe due to the transparency of both the coating and the substrate. The size of the etched zones was observed to grow linearly with etching time, so that extrapolation to zero time enabled the defect population of the coating to be determined $[4,16,17]$.

\subsection{Internal stresses measurements}

The in-plane internal stress state in as-grown $\mathrm{SiO}_{x}$ coatings was calculated from the radii of curvature of the film before, $R_{1}$, and after, $R_{2}$, coating deposition, following the analysis of Röll [17]:

$\sigma_{i}=-\frac{E_{\mathrm{s}} h_{\mathrm{s}}^{2}}{6\left(1-v_{\mathrm{s}}\right) h_{\mathrm{c}}}\left(1+\frac{h_{\mathrm{c}}}{h_{\mathrm{s}}}\left(4 \frac{E_{\mathrm{c}}}{E_{\mathrm{s}}}-1\right)\right) \cdot\left(\frac{1}{R_{2}}-\frac{1}{R_{1}}\right)$

where $E_{\mathrm{s}}$ and $E_{\mathrm{c}}$ are the Young's moduli of the substrate and coating, respectively equal to $4.8 \mathrm{GPa}$ and $79.5 \mathrm{GPa}$ [18], $v_{\mathrm{s}}$ is the substrate Poisson's ratio, and $h_{\mathrm{s}}$ and $h_{\mathrm{c}}$ are the corresponding thickness. The usual convention, where compressive stresses are negative, was adopted. The radii $R_{1}$ and $R_{2}$ of samples supported freely on two vertical aluminum plates were measured with a binocular lens (Olympus SZH). The internal stress of the $\mathrm{SiO}_{x}$ coatings was found to be compressive and smaller than $200 \mathrm{MPa}$, a negligible value compared to the coating cohesive strength, which is equal to several GPa as will be demonstrated later. Internal stresses will thus be disregarded in further analyses of the cohesive and adhesive properties of the $\mathrm{SiO}_{x} / \mathrm{PET}$ materials.

\subsection{In-situ fragmentation testing in a SEM}

Coating cohesive properties and coating/substrate interfacial shear strength were determined by means of fragmentation tests carried out in-situ in a scanning electron microscope. The method, developed for the specific case of ultrathin dielectric coatings was described in details elsewhere [15], and a summary is given here. Gold-coated samples were clamped in a miniature tensile testing device and prestrained to $0.2 \pm 0.1 \%$ strain. The tensile unit was mounted in a JEOL JSM-6300F scanning electron microscope, and the sample was loaded stepwise up to predefined nominal strain levels, $\varepsilon$. The effect of the charge dissipating gold layer on the failure of the underlying oxide film was carefully calibrated to avoid the creation of artifacts. The occurrence of damage in the oxide under tensile load was analyzed in terms of crack onset strain (COS) and crack density, defined as the inverse of the average fragment length. The crack density was then averaged over eight micrographs for the initial fragmentation stages, and over three micrographs for the largest strains.

The early stages of the fragmentation process were analyzed following a weakest link approach, assuming a Weibull-type size dependent probability of failure [19], from which was derived the size-dependent coating cohesive strength [20]:

$\sigma_{\max }(l)=\beta\left(l / l_{0}\right)^{-1 / \alpha} \Gamma(1+1 / \alpha)$

where $l$ is the size of a coating element, $l_{0}$ is a normalization factor, chosen equal to $1 \mu \mathrm{m}, \Gamma$ is the gamma function, and $\alpha$ and $\beta$ are the Weibull shape and scale factors, respectively. For thin coatings, these two factors are usually derived from a linear approximation of the initial part of the fragmentation diagram, where the evolution of the average fragment size is reported as a function of applied strain, in logarithmic coordinates [20].

The advanced stage of the fragmentation process, when fragment size reaches a saturation regime controlled by interfacial stress transfer was analyzed following the perfectly plastic Kelly-Tyson model [21]. In spite of its simplicity, such treatment is quite robust, essentially because of the high elastic contrast between the oxide and the polymer [22]. Solving the force balance on a small coating element leads to the determination of the interfacial shear strength (IFSS):

$\tau=2 h_{\mathrm{c}} \sigma_{\max }\left(l_{\mathrm{c}}\right) / l_{\mathrm{c}}$

where $\tau$ is the IFSS, characteristic of the coating/substrate adhesion, $h_{\mathrm{c}}$ is the coating thickness, $\sigma_{\max }$ its tensile strength, characteristic of its cohesion, and $l_{\mathrm{c}}$ is the critical stress transfer length defined as the minimum coating length in which the maximum allowable coating stress $\left(\sigma_{\max }\right)$ is achieved. The length $l_{\mathrm{c}}$ is not a measurable quantity, and was related to the average fragment length at saturation, $\bar{l}_{\text {sat }}$, that is, the average length when an increase in strain does not lead to further fragmentation of the coating: $\bar{l}_{\text {sat }}=0.67 \cdot l_{\mathrm{c}}[23]$.

\section{Results}

\subsection{Atomic oxygen etching}

The size distributions of the defects in the oxide layer on PET with and without additives are reported in Fig. 2, together with a log-normal distribution fit. It is important to note that the atomic oxygen etching method enabled at best the detection of defects of size larger than several tens of 
(a)

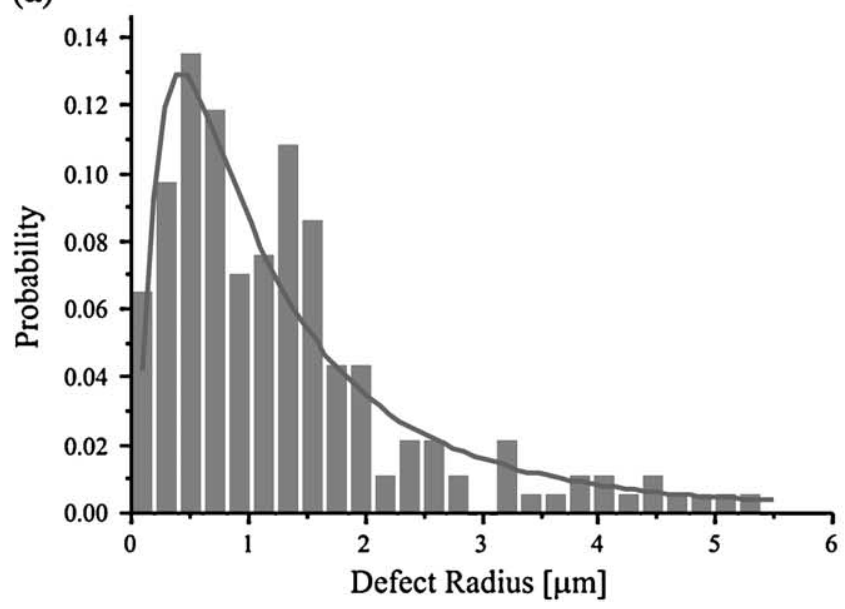

(b)

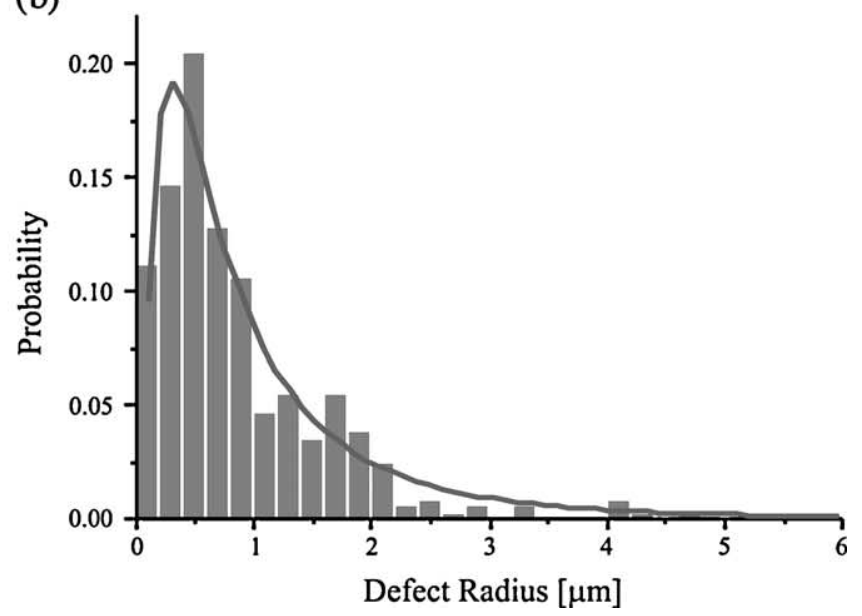

Fig. 2. Defect population of the $10 \mathrm{~nm}$ thick $\mathrm{SiO}_{x}$ coating deposited on PET substrates without additives (a) and with additives (b).

nanometers. Smaller defects, related to the nanoscale disordered structure of the oxide, were not detected with this method. The nanometric additives led to a marked increase in the defect density (from $179 \mathrm{~mm}^{-2}$ for PET without additives to $356 \mathrm{~mm}^{-2}$ for PET with additives), and to a decrease of the mean defect size (from $1.06 \mu \mathrm{m}$ for PET without additives to $0.72 \mu \mathrm{m}$ for PET with additives). In other words, such additives influenced the plasma deposition process by creating submicrometric defects of a few hundreds of nanometers in radius.

\subsection{Fragmentation of the $\mathrm{SiO}_{x}$ coating}

The progressive damage in the $10 \mathrm{~nm}$ thick $\mathrm{SiO}_{x}$ coating on PET films without and with additives is represented in

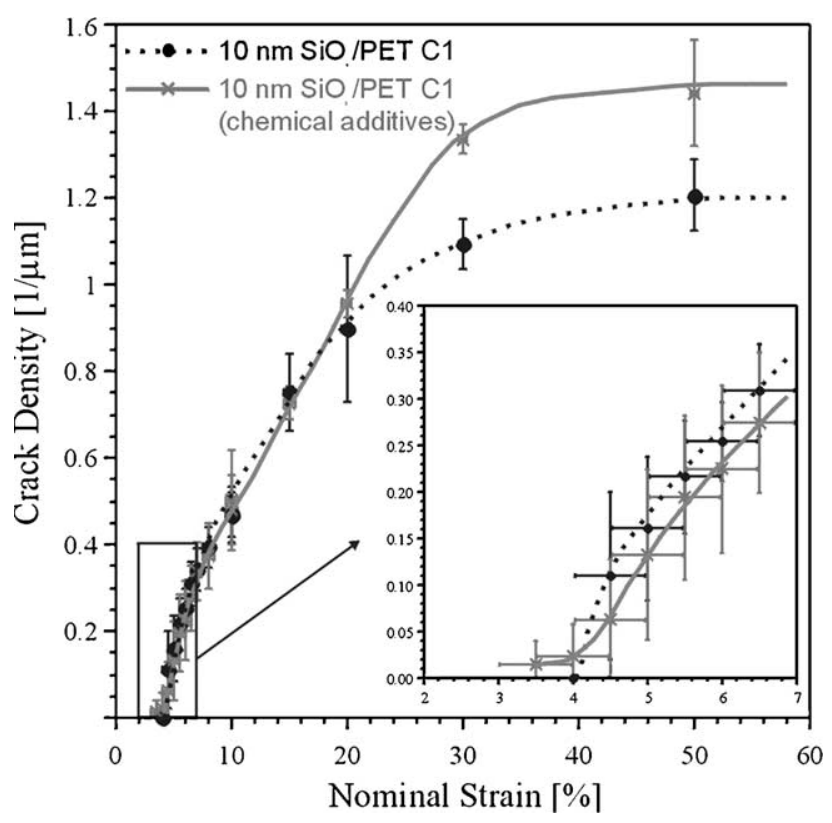

Fig. 3. Crack density vs. nominal strain for a $10 \mathrm{~nm}$ thick $\mathrm{SiO}_{x}$ coating on PET substrates without and with additives.
Fig. 3 in terms of crack density vs. strain, measured in-situ in the SEM. The crack onset strain was decreased by $20 \%$ in the presence of additives, from $4.0 \pm 0.3 \%$ to $3.3 \pm 0.3 \%$. The saturation crack density was increased from $1.2 \mu \mathrm{m}^{-1}$ for the $\mathrm{SiO}_{x} / \mathrm{PET}$ film without additives to $1.45 \mu \mathrm{m}^{-1}$ for the $\mathrm{SiO}_{x} / \mathrm{PET}$ film with additives (Table 2).

\section{Discussion}

\subsection{Coating cohesive strength and coating/substrate interfacial shear strength}

The mechanical properties of the coated film are summarized in Table 3, with the Weibull parameters and the characteristic stress transfer lengths. The additives led to an increase of the shape parameter $\alpha$ (from 3.3 for the film without additives to 6.1 for the film with additives) and a decrease of the scale parameter $\beta$ (from $6.9 \mathrm{GPa}$ for the film without additives to $5.7 \mathrm{GPa}$ for the film with additives). Additives induced a narrowing of the Weibull distribution, and a decrease of the average coating strength, hence of the oxide strain to failure. The latter is due to the modification of the defect population of the coating in presence of additives, and is discussed in the following section.

The higher crack density at saturation observed in the case of a PET film containing additives is a further demonstration of the decrease of the average cohesive strength of the coating, and not a result of a lower

Table 2

Density and average radius of the defects in a $10 \mathrm{~nm}$ thick $\mathrm{SiO}_{x}$ coating deposited on PET substrates without and with additives

\begin{tabular}{lll}
\hline $\mathrm{SiO}_{x} /$ PET film & $\begin{array}{l}\text { Defect density } \\
{\left[\mathrm{mm}^{-2}\right]}\end{array}$ & $\begin{array}{l}\text { Average radius } \\
\text { of defects }[\mu \mathrm{m}]\end{array}$ \\
\hline Without additives & 179 & $1.06 \pm 0.29$ \\
With additives & 356 & $0.72 \pm 0.16$ \\
\hline
\end{tabular}


Table 3

Micromechanical and Weibull parameters of $\mathrm{SiO}_{x}$ coatings on PET films without and with additives

\begin{tabular}{lllllllr}
\hline $\mathrm{SiO}_{x} / \mathrm{PET}$ film & Crack onset strain $[\%]$ & $\bar{I}_{\text {sat }}[\mu \mathrm{m}]$ & $l_{\mathrm{c}}[\mu \mathrm{m}]$ & $\alpha$ & $\beta[\mathrm{GPa}]$ & $\sigma_{\max }\left(l_{\mathrm{c}}\right)[\mathrm{GPa}]$ & $\mathrm{IFSS}[\mathrm{MPa}]$ \\
\hline Without additives & $4.0 \pm 0.5$ & $0.83 \pm 0.06$ & $1.24 \pm 0.08$ & 3.3 & 6.9 & 5.8 & 94 \\
With additives & $3.3 \pm 0.5$ & $0.69 \pm 0.06$ & $1.04 \pm 0.09$ & 6.1 & 5.7 & 5.2 & 101 \\
\hline
\end{tabular}

adhesion between the coating and the substrate. The interfacial shear strength (IFSS) between the oxide coating and the polymer substrate is indeed marginally increased by the presence of additives in the superficial PET layers. Moreover, in both cases, the IFSS is much larger than the bulk shear stress at yield of the substrate (approx. 50 $\mathrm{MPa}$ ). Such very high levels of adhesion suggest that the behavior of the interfacial region is strain hardening and therefore prevents delamination [20], thanks to a high density of covalent bonds formed during plasma deposition [6].

\subsection{Effect of coating defects on cohesive strength}

In previous sections, it was found that the additives located in the superficial layers of the polymer substrate doubled the coating defect density and reduced its crack onset strain and cohesive strength. In order to analyze the relation between coating defect density and cohesive strength, a model was developed based on a study of $\mathrm{Wu}$ and Cheng [24]. The present approach considers two perfectly bonded layers (Fig. 4), with an upper layer $\left(\mathrm{SiO}_{x}\right)$ containing a perfectly cylindrical hole, and a homogeneous bottom layer (PET). This approach enables the stress concentration generated by a density of non-interacting holes to be calculated.

The stresses and displacements were decomposed in Taylor series, and second-order terms in the $z$ coordinate of the $x-y$ plane displacements $\mathrm{u}$ and $\mathrm{v}$ were considered to satisfy all the compatibility requirements for the multilayer composite. The materials were assumed elastic and isotropic. $\mathrm{Wu}$ and Cheng gave a solution of the above equations by developing the stress potentials in Laurent series for the two plates of the system [24]. The coefficients

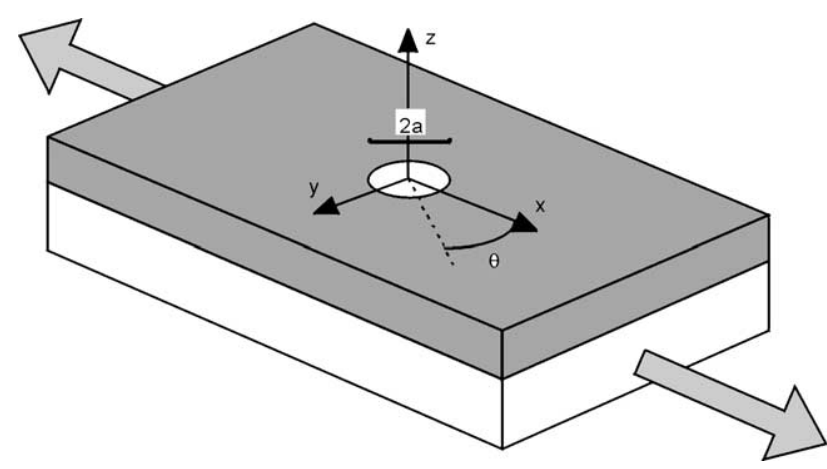

Fig. 4. Thin bilayered composite with an upper layer containing a hole. of the Laurent series, $\left\{c_{m}\right\}_{1}^{\infty}$, were determined by the condition of displacement continuity of the plate with the hole and the patching plate at the interface, expressed by $\left(u_{\mathrm{c}}+i v_{\mathrm{c}}\right)=\left(u_{\mathrm{s}}+i v_{\mathrm{s}}\right)$ with, assuming $h_{\mathrm{c}} \ll a$ :

$$
\begin{aligned}
2 \cdot G_{\mathrm{c}} \cdot\left(u_{\mathrm{c}}+i \cdot v_{\mathrm{c}}\right)= & \frac{S_{\mathrm{c}}}{4}\left\lfloor\left(\kappa_{\mathrm{c}}-1\right) \cdot \xi+2 \cdot \bar{\xi}+2 \cdot a^{2} \cdot\right. \\
& \left.\times\left(\frac{\kappa_{\mathrm{c}}}{\xi}+\frac{1}{\bar{\xi}}+\frac{\xi}{\bar{\xi}^{2}}-\frac{a^{2}}{\bar{\xi}^{3}}\right)\right\rfloor-\frac{1}{2} \cdot \sum_{m, \mathrm{odd}} c_{\mathrm{m}} \cdot \\
& \times\left(\frac{1}{\xi^{m}}+\frac{m \cdot \xi}{\bar{\xi}^{m+1}}-\frac{m \cdot(m+1)}{m+2} \cdot \frac{a^{2}}{\bar{\xi}^{m+2}}\right)
\end{aligned}
$$

and:

$$
\begin{aligned}
2 \cdot G_{\mathrm{s}} \cdot\left(u_{\mathrm{s}}+i \cdot v_{\mathrm{s}}\right)= & \frac{S_{\mathrm{S}}}{4} \cdot\left[\left(\kappa_{\mathrm{s}}-1\right) \cdot \xi+2 \cdot \bar{\xi}\right]-\frac{1}{2} \cdot\left(\frac{h_{\mathrm{c}}}{h_{\mathrm{s}}}\right) \cdot \sum_{m \text { odd }} c_{m} \\
& \times\left(\frac{1}{\xi^{m}}+\frac{\kappa_{\mathrm{s}} \cdot m \cdot \xi}{\bar{\xi}^{m+1}}-\frac{\kappa_{\mathrm{s}} \cdot m \cdot(m+1)}{m+2} \cdot \frac{a^{2}}{\bar{\xi}^{m+2}}\right)
\end{aligned}
$$

where $u_{\mathrm{c}}$ and $v_{\mathrm{c}}$ represent the spatial displacements of the coating in the $x$ and $y$ directions, respectively, and $u_{\mathrm{s}}$ and $v_{\mathrm{s}}$ the corresponding spatial displacements of the substrate. The factors $G_{j}$ and $S_{j}$ are the shear modulus and far-field stress, respectively, $\kappa_{j}=\left(3-v_{j}\right) /\left(1+v_{j}\right), v_{j}$ being the Poison's ratio, with $j=\mathrm{c}, \mathrm{s}$ for the coating and substrate, respectively. The term $\xi=x+i y$ is a complex variable. The above set of equations was solved numerically using Fortran77 and a discrete mesh of the complex plane, in order to determine the $\left\{c_{m}\right\}_{1}^{\infty}$ series. Due to the circular geometry of the model, this was done in the $(r ; \theta)$ polar configuration. A uniform 1400-point mesh was considered for the $\theta$ coordinate from 0 to $\pi / 2$, and a geometrical series 1400-point mesh was considered for the $\mathrm{r}$ coordinate from 0 to $10 \mathrm{a}$. The $r=10 a$ limit was set following the St-Venant principle, which states that the presence of a defect of characteristic size $a$, has an effect on the stress state on a region of characteristic size $5 \mathrm{a}$. This limit was verified by the present simulations, corresponding to an upper limit for the hole density equal to approx. $7000 \mathrm{~mm}^{-2}$. After a check of convergence, the $\left\{c_{m}\right\}_{1}^{\infty}$ series was truncated to the 5th order in order to render the simulation possible. The stress concentration in the straining direction $x$ was then obtained from the stress potentials following the development of $\mathrm{Wu}$ and Cheng. 


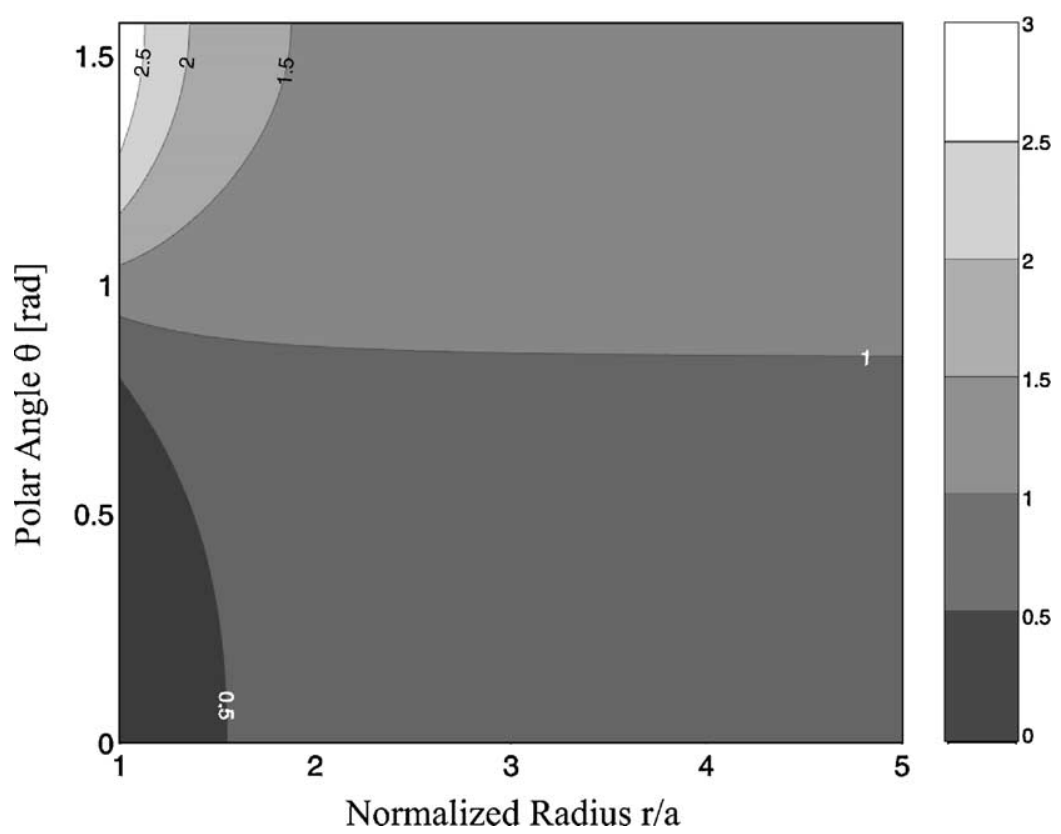

Fig. 5. Polar representation of the stress concentration induced by a hole in a layer patched to another intact layer

Fig. 5 shows a polar representation of the stress concentration in the straining direction $x$ as a function of the coordinates $r$ and $\theta$. The Poisson's ratio, Young's modulus and thickness of the top layer with the hole $\left(\mathrm{SiO}_{x}\right)$ and of the bottom layer (PET) were chosen as $v_{\mathrm{c}}=0.2$ [25], $E_{\mathrm{c}}=80 \mathrm{GPa}, h_{\mathrm{c}}=12 \mathrm{~nm}, v_{\mathrm{s}}=0.45[26], E_{\mathrm{s}}=4 \mathrm{GPa}$, and $h_{\mathrm{s}}=12 \mu \mathrm{m}$. The hole radius was set equal to $1 \mu \mathrm{m}$, according to the oxygen atomic etching results obtained in the previous section. The maximal stress concentration is found as expected at the equator of the circular defect and is exactly equal to 3 , which reproduces the well-known case of a drilled plate without a patching plate [10].

In order to take into account the presence of circular defects in the $\mathrm{SiO}_{x}$ coating, the stress concentration induced by the hole (Fig. 5) was inserted into the Weibull strength distribution equation, and the fracture probability for an applied stress $\sigma$ was then deduced. By reference to the weakest link model [27], the cumulative distribution function for the whole coating $G(\sigma)$ is:

$$
\begin{aligned}
G(\sigma) & =1-\prod_{k}\left[1-F_{k}(\sigma)\right]^{A(k)} \\
& =1-\prod_{k} \exp \left[-\frac{l}{l_{0}} \cdot\left(\frac{\sigma_{k}}{\beta}\right)^{\alpha}\right]^{A(k)}
\end{aligned}
$$

where the product is done on each mesh, $k$, of area $A(k)$. It is important to point out that such an approach is only valid in case of non-interacting holes. Moreover, the stress concentration at a distance from the centre of the hole larger than 10 times the hole radius is considered to be negligible, and equal to one (i.e., the stress is equal to the far-field stress).

In order to determine the effect of the defect density on the coating mechanical strength, the cumulative distribution function $\mathrm{G}(\sigma)$ for an intact coating at a gauge length of 100 $\mu \mathrm{m}$ was compared to that of a system whose coating has the same mechanical properties (i.e., same Weibull shape factor $\alpha$ ) but which is embrittled by a homogeneous distribution of circular defects of radius $1 \mu \mathrm{m}$, and of density ranging from 0 to 2500 defects $/ \mathrm{mm}^{2}$. The function $G(\sigma)$ was calibrated using the properties of the $\mathrm{SiO}_{x} / \mathrm{PET}$ film without additives (i.e., $\alpha=3.3$ and $\beta=6.9 \mathrm{GPa}$ ). The evolution of the scale parameter $\beta$ as a function of the density of circular defects is given in Fig. 6, and compared to the experimental results for the $\mathrm{SiO}_{x} / \mathrm{PET}$ films.

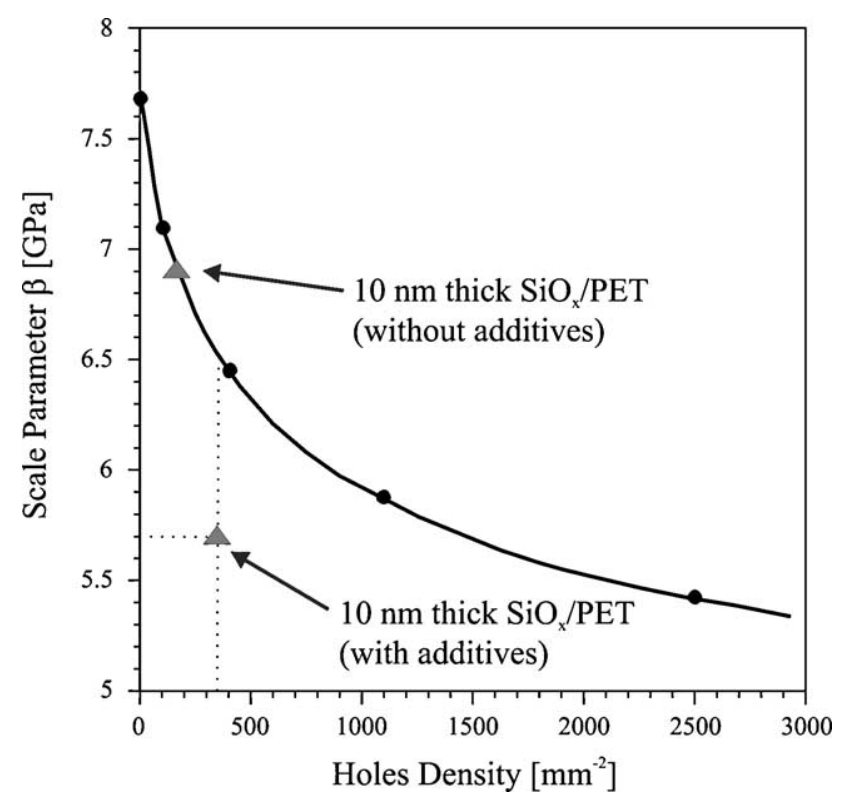

Fig. 6. Effect of circular holes on the $\beta$ Weibull scale parameter. The triangles correspond to experimental data and the solid line to the theoretical calculation. 
The theoretical decrease of the scale parameter with increasing defect density provides an upper bound to the experimental decrease, since real defect are not perfectly circular, and therefore are associated with higher stress concentrations. Nevertheless, it is evident that circular defects have a marked influence on the cumulative strength distribution function and decrease the tensile strength of the $\mathrm{SiO}_{x}$ coating by increasing the probability of failure for a given stress applied to the composite film. As long as the limitations of the model are not reached (hole density lower than $7000 \mathrm{~mm}^{-2}$ ), the important parameter is the hole density, and not their size. This should be compared with the results obtained for gas permeation by Rossi and Nulman, who showed that for permeation, small holes are much more detrimental than large ones, for an equivalent hole surface [9]. In view of the present mechanical analysis, it is therefore essential to control both the density of nanometric defects in order to guarantee low permeation, and the density of micrometric defects in order to improve the cohesive properties of the barrier layer.

\section{Conclusions}

The influence of process additives located in the superficial layers of PET substrates on the cohesion and adhesion of thin plasma-deposited oxide films was investigated by means of uniaxial fragmentation tests in-situ in a SEM. The defect population was determined using a reactive ion etching technique, and theoretical calculations were carried out to determine the effect of defect-induced stress concentrations in the coating. It was found that:

The presence of additives in the superficial layers of PET substrates doubled the defect density in the oxide coating and led to a $20 \%$ decrease of coating strain to failure. This was due to a decrease in the average strength of the coating resulting from stress concentrations at microdefects, as shown by calculations based on a Weibull probability of failure;

The shear strength of the $\mathrm{SiO}_{x} / \mathrm{PET}$ interface was not affected by substrate additives, and was larger than the substrate yield shear stress, which suggested that a high density of covalent bonds were formed at the coating/ polymer interface during plasma deposition.

\section{Acknowledgements}

The authors would like to thank Tetra Pak (Suisse) SA and the Top Nano 21 initiative of the Swiss Commission for Technology and Innovation (CTI) for financial support, and the Centre Interfacultés de Microscopie Electronique (CIME) of the Ecole Polytechnique Fédérale de Lausanne (EPFL) for technical assistance during the SEM observations.

\section{References}

[1] H. Chatham, Surf. Coat. Technol. 78 (1996) 1.

[2] P.E. Burrows, G.L. Graff, M.E. Gross, P.M. Martin, M.K. Shi, M. Hall, E. Mast, C. Bonham, W. Bennett, M.B. Sullivan, Displays 22 (2001) 65 .

[3] A.P. Roberts, B.M. Henry, A.P. Sutton, C.R.M. Grovenor, G.A.D. Briggs, T. Miyamoto, A. Kano, Y. Tsukahara, M. Yanaka, J. Membr. Sci. 208 (2002) 75.

[4] A.S.D. Sobrinho, G. Czeremuszkin, M. Latreche, M.R. Wertheimer, J. Vac. Sci. Technol., A 18 (2000) 149.

[5] G. Rochat, Y. Leterrier, J.-A.E. Månson, P. Fayet, Surf. Coat. Technol. 174-175 (2003) 1029.

[6] Y. Leterrier, Prog. Mater. Sci. 48 (2003) 1.

[7] G. Rochat, A. Delachaux, D. Favez, Y. Leterrier, J.-A.E. Månson, P. Fayet, Surf. Interface Anal. 35 (2003) 948.

[8] G. Rochat, Y. Leterrier, C.J.G. Plummer, J.-A.E. Månson, R. Szoszkiewicz, A.J. Kulik, P. Fayet, J. Appl. Phys. 95 (2004).

[9] G. Rossi, M. Nulman, J. Appl. Phys. 74 (1993) 5471.

[10] S.P. Timoshenko, J.N. Goodier, Theory of Elasticity, 3rd ed., McGraw Hill, Singapore, 1970.

[11] J.H. Briston, Plastic Films, 3rd edition, Longman Scientific and Technical, Harlow, Essex, England, 1989.

[12] M.B. Khan, C. Keener, Polym. Eng. Sci. 36 (1996) 1290.

[13] J.S.G. Ling, G.J. Leggett, Polymer 38 (1997) 2617.

[14] B.D. Beake, J.S.G. Ling, G.J. Leggett, Polymer 41 (2000) 2241

[15] G. Rochat, Y. Leterrier, J.-A.E. Månson, P. Fayet, Thin Solid Films 437 (2003) 204.

[16] A.D. Sobrinho, G. Czeremuszkin, M. Latreche, M.R. Wertheimer, Appl. Phys., A Mater. Sci. Process. 68 (1999) 103.

[17] K. Röll, J. Appl. Phys. 47 (1976) 3224.

[18] Y. Leterrier, P. Sutter, J.-A.E. Månson, J. Adhesion 69 (1999) 13.

[19] W. Weibull, J. Appl. Mech. 18 (1951) 293.

[20] Y. Leterrier, L. Boogh, J. Andersons, J.-A.E. Månson, J. Polym. Sci., B, Polym. Phys. 35 (1997) 1449.

[21] A. Kelly, W.R. Tyson, J. Mech. Phys. Solids 13 (1965) 329.

[22] C.Y. Hui, D. Shia, L.A. Berglund, Compos. Sci. Technol. 59 (1999) 2037.

[23] Y. Leterrier, Y. Wyser, J.-A.E. Månson, J. Hilborn, J. Adhesion 44 (1994) 213

[24] X.J. Wu, S.M. Cheng, J. Appl. Mech. Trans. ASME 66 (1999) 95.

[25] G. Carlotti, L. Doucet, M. Dupeux, Thin Solid Films 296 (1997) 102.

[26] R. Feng, R.J. Farris, J. Appl. Polym. Sci. 86 (2002) 2937.

[27] W.A. Fraser, F.H. Achker, A.T. DiBenedetto, Proc. 30th Conf. SPI Reinforced Plastics Division, The Society of the Plastics Industry, 1975 . 